\title{
Effect of a Clinical Pathway on Outcome of Children with Cancer Suffering from Neutropenia Undergoing Chemotherapy Treatment
}

Yasmen Adel Mohammed: Master of Pediatric Nursing, Faculty of Nursing, Benha University

Rahma Soliman Bahgat: Professor of Pediatric Nursing, Faculty of Nursing, Tanta University

Hala Abdel Badie Nayel: Professor of Pediatric Medicine, Cairo University

Sabah Mohamed El sayed: Lecturer of Pediatric Nursing, Faculty of Nursing, Tanta University

\section{Abstract}

The child suffering from neutropenia undergoing chemotherapy is demanding and requiring the integration of skills from numerous different specialties. These children often have prolonged hospitalizations, which may be marked by many complications. The aim of this study to design, implement and evaluate the effect of a clinical pathway for children with neutropenia undergoing chemotherapy.Quasi experimental research design was used. The study was carried out at Tanta Cancer Center Affiliated to the Ministry of Health. Out patient Clinic of Oncology and Inpatient Pediatric Oncology Department in Benha Children Hospital. 72 children with neutropenia undergoing chemotherapy was recruited for the study. Four tools were used for data collection, questionnaire sheet, Nutritional Assessment Sheets, Complication monitor sheet and follow pathway design for the study group. The result of the study revealed that there was no statistically significant difference between the study and control groups on admission but there was a statistically significant difference between the study and control groups after the implementation of the clinical pathway for the study group compared with the routine hospital policy care. Conclusion: Children with neutropenia undergoing chemotherapy to whom clinical pathway was applied fewer complications, less hospital stay and less readmission than those who received routine hospital care. Recommendations: Clinical pathway should be integrated into the routine nursing care for children with neutropenia undergoing chemotherapy.

Key words: Neutropenia, Chemotherapy, Prolonged Hospitalization, Clinical Pathway 


\section{Tanta Scientific Nursing Journal}

\section{Introduction}

Cancer in children and adolescents is a life-altering event for them as well as their families. Although advances in treatment have increased the overall 5-year survival rate for childhood cancers to approximately $80 \%$, cancer is still the second leading cause of death following accidents in children aged 5 to 14 years, however, little evaluation of the overall experience at the end of life of children who are dying of cancer or of their symptoms other than pain, cancer begins when cells in a part of the body start to grow out of control ${ }^{(14)}$

Cancer, an abnormal growth of cells which tend to proliferate in an uncontrolled way and metastasize. Cancer is not one disease. It is a group of more than 100 different and distinctive diseases. Cancer can involve any tissue of the body and have many different forms in each body area. Most cancers are named for the type of cell or organ in which they start ${ }^{(15)}$

Cancer is a generic term for a large group of diseases that can affect any part of the body. Other terms used are malignant tumors and neoplasms cancer is the creation of abnormal cells that grow beyond their usual boundaries, and which can then invade adjoining parts of the body and spread to other organs. This process is referred to as metastasizing. Metastases are the major cause of death from Cancer. ${ }^{(1)}$ Childhood cancer in Egypt is a growing concern for the society since its incidence has been increasing rapidly. The high mortality rate is assumed, due to the inadequate access to medical care in developing countries as there are very few hospitals or centers existing in these countries with the children cancer hospital being an exception to the rule. Lack of education and knowledge of health concerns particularly relating to children have delayed many families from seeking medical help and treatment. In addition, lack of transportation for the children obstacles concerning transportation from rural to urban places makes the available health care inaccessible. ${ }^{(34)}$

Leukemia is the most common type of childhood cancer, representing about one third of all cancers in children less than 15 years of age. Leukemia is a condition where too many underdeveloped white blood cells are found in the blood and bone marrow. Most of childhood leukemia's are acute lymphatic leukemias (ALL); other types include acute myeloid leukemia (AML) and chronic myeloid leukemia (CML). Brain tumors are the most common solid tumors in childhood ${ }^{(3)}$ Chemotherapy is a chemical that binds to 
and specifically kills tumor cells. Chemotherapy is usually systemic treatment, meaning that the drugs flow through the bloodstream to nearly every part of the body most cancer chemotherapeutic drugs are given intravenous or intramuscular. Some anticancer agents are taken orally. ${ }^{(4)}$

Side Effects of chemotherapy depend mainly on the drugs and the doses the child receives. Most anticancer drugs affect cells that divide rapidly. These include blood cells, which fight infection, help the blood to clot, or carry oxygen to all parts of the body. When blood cells are affected by anticancer drugs, children are more likely to develop infections, may bruise or bleed easily, and may have less energy. Cell hat line the digestive tract also divide rapidly resulting, loss of appetite, nausea and vomiting As a result of chemotherapy, child has side effects, such as, hair loss, or mouth sores. ${ }^{(7)}$

Cytotoxic chemotherapy suppresses the hematopoietic system, impairing host protective mechanisms so limiting the doses of chemotherapy that can be tolerated should be done. Neutropenia, the most serious hematologic toxicity, is associated with the risk of life-threatening infections as well as chemotherapy dose reductions and delays that may compromise treatment outcomes. ${ }^{(8)}$
Neutropenia is a condition where there are abnormally low levels of neutrophils in the blood supply. Neutrophils are an important type of white blood cell, vital for fighting off pathogens, particularly bacterial infections. Neutropenia can be caused by a decrease in neutrophils production, accelerated usage of neutrophils, increased destruction of neutrophils or a combination of all three factors. Neutropenia can be temporary (acute) or long-lasting .The condition is also split into congenital (present from birth) and acquired neutropenia (develops later in life). ${ }^{(105)}$

Neutropenic child their ability to mount an inflammatory response is limited. There are often few signs and symptoms of infection except for fever. Localized infections that appear minor can rapidly progress to a systemic infection, which may be fatal, therefore, considered a serious and potentially life-threatening complication in children undergoing systemic chemotherapy ${ }^{(9)}$

Clinical pathway is a strategy that reduces resource utilization while maintaining the quality of care. The most popular methods intended to meet intense pressures to reduce the costs of health care. Clinical pathways are management plans that display goals for children and provide the corresponding ideal sequence and timing 
of staff actions to achieve those goals with optimal efficiency. ${ }^{(11)}$

Clinical pathway is a tool for achieving coordinated care and desired health outcomes within an anticipated time frame, by using the appropriate resources available. A clinical pathway is a blueprint that guides the clinician in the provision of care $^{(13)}$

A clinical pathway is a method for the child -care management of a well-defined group of children during a well-defined period of time. A clinical pathway explicitly states the goals and key elements of care based on Evidence Based Medicine (EBM) guidelines, best practice and patient expectations by facilitating the communication, coordinating roles and sequencing the activities of the multidisciplinary care team, children and their relatives; by documenting, monitoring and evaluating variances; and by providing the necessary resources and outcomes. The aim of a clinical pathway is to improve the quality of care, reduce risks, increase children satisfaction and increase the efficiency in the use of resources and decrease length of hospitalization. ${ }^{(164)}$

\section{Aim of the Study}

The aim of the study was to determine the effect of clinical pathway on outcome of children with cancer suffering from neutropenia undergoing chemotherapy through. Design clinical pathway for children with cancer suffering from neutropenia undergoing chemotherapy Evaluate the effectiveness of clinical pathway on outcome of children with cancer suffering from neutropenia under going chemotherapy.

\section{Research hypothesis}

Children with cancer suffering from neutropenia undergoing chemotherapy treatment whom clinical pathway is expected to applied have fewer complications, less hospital stay ,less readmission and enhanced quality of care than those who will receive routine hospital care.

\section{Materials and Method}

\section{Research Design:}

A quasi -experimental research design was utilized in this study

\section{Settings:}

The study was carried out at Tanta Cancer Center Affiliated to the Ministry of Health (Pediatric Oncology department) - Out patient Clinic of Oncology and Inpatient Pediatric Oncology Department in Benha Children Hospital

\section{Subject:}

The subject of the study was consist of (72) children with cancer were selected from previously mention setting. The sample was randomly selected and divided into two equal groups. 
Study group consist of 36 children were exposed to clinical pathway by the researcher, divided to Control group consist of 36 children were exposure to routine hospital care.

\section{Tools of data collection:}

Four tools were developed and used by researcher after reviewing the literature to collect information about.

\section{Tool I:A questionnaire sheet}

\section{Part I- Bio socio demographic data}

Data related to child: such as age, sex, birth order, level of education. This tool will be design by researcher in simple Arabic language after reviewing related literature.

\section{Part II -Medical History:}

Medical history was designed by the hospital policy (patient file), to identify eligible criteria for clinical pathway implementation, researcher will search for present diagnosis, main complain, past medical history and number of hospital admission for cycles of chemotherapy, cumulative dose toxicity on bone marrow of most chemotherapy treatment.

Part III-Dietary profile for the child included:

Feeding habits of the child such as, number of meals per day, their appetites, likes and un likes.

Feeding problem such as, nausea, vomiting, loss of appetite, difficulty in chewing and swallowing food, dryness of the mouth and change of food taste.

Part IV: Daily living activity as hyper activity, normal and limited activity, child play or not, types of play (quite play, active play, mixed play) and hours of play during the day.

\section{Tool II: Nutritional Assessment Sheets:}

\section{It includes three parts:}

Part I: Physical assessment sheet of cancer child which included:

Observation for general condition from head to toes includes hair, face, eye, lips, gume, skin, tounge, any gastro-intestinal tract problem, muscular-skeletal system and measurement of vital signs (temperature, pulse, respiration, blood pressure).

\section{Part II: Anthropometric measurements}

\section{sheet which includes:}

Weight, height, body mass index, upper arm circumference and triceps skin fold thickness. Each of these measurements will be taken according to the standard procedure recommended by Jelliffe. (177)

Weight/age will be measured using bathroom scale and recorded to the nearest tenth of a kilogram. It is used as indicator of the nutrional status for the children.

Height /age will be measured and taken to the nearest $0.1 \mathrm{~cm}$ and then recorded.

Body Mass Index (BMI) will be calculated as follow: 
$\mathrm{BMI}=$ Weight in $\mathrm{KG} /($ height in meter $) 2$..

Mid arm circumference will be measured by placing the tape gently but firmly around the left upper am while hanging freely and on its mid point.The measurements will be recorded to the nearest $0.1 \mathrm{~cm}$.

Skin -fold thickness: Triceps skin -fold thickness will be measured using harpenden caliper.

\section{Part III: Laboratory investigations:}

-Laboratory investigation

included.Complet blood count.Renal function test. Liver function test.Lactate Dehydrogenises.Earthrocyte sedimentation rate. And Bone marrow aspiration.

Part IV: Daily dietary intake : to estimate the caloric intake by 24 hours recall method and fluid intake per day according to the food exchange list including the five main groups (milk group, meat, fruit, vegetable, bread, cereal. it will be calculated by number serving \ day and the average will be determined

\section{Tool III: Complication monitor sheet:}

This sheet will be designed by researcher for monitoring the presence of complication. It monitor complications which cover general and specific complications that resulting from administrating protocols of cycles chemotherapy. General complication included, pancytopenia, (bone marrow depression) ,Infection, anorexia, loss of weight, fever, mucositis, vomiting, diarrhea, hair loss and extra vacation. Specific complication included central nervous system as headache, muscle skeletal system as boneach, bleeding, cardiac complication as tachycardia and respiratory complication as tachyapnea.

\section{Clinical pathway designed for children:}

\section{IV: Tool}

The clinical pathway out come was designed by the researcher, implemented and evaluated for the clinical pathway included following items: -Feeding habit and problems. Daily living activity. Nutritional assessment sheet of cancer child .Physical assessment sheet of cancer child Anthropometric measurement Laboratory investigations Daily dietary intake

4-Safety measures for Medications intake which include the following:Blood transfusion (taken, not taken) Platelet intake (taken, not taken) .Antibiotic intravenous (oral, single, double) .Antipyretic (taken, not taken).Chemotherapy (taken, not taken) .Growth factors (taken, not taken) .Steroids: (taken, not taken)

\section{Ethical and legal considerations}

privacy and confidentiality was protected. Children were reassured that the obtained information were confidential and used 
only for purpose of the study. Confidentiality and privacy were assured by code number on sheets instead name, Withdraw from the study was reserved at any time.

\section{Procedure}

The actual field work was carried out from May 2015 up to November 2015 for data collection. The researcher were available in the study settings four day per week, on Saturday and Tuesday at the mooring shift from 8.00 Am to 2.00 P.m in Out patient Clinic of Oncology and Inpatient Pediatric Oncology Department in Benha Children Hospital. and on Sunday and Wednesday in Tanta Cancer Center Affiliated to the Ministry of Health (Pediatric Oncology department), the researcher introduces herself to all hospital staff members in the two hospitals settings. The researcher explained the aim of the study and asked for the hospital team for cooperation. Children with cancer suffering from neutropenia undergoing chemotherapy were divided into two equal groups (study and control group). The researcher performs the research in the following phase:

\section{1- Assessment phase:}

Assessment of both study and control group of children with cancer suffering from neutropenia , socio demographic data, medical history, Dietary profile for the child Daily living activity nutritional assessment included, Physical assessment sheet of cancer child, Anthropometric measurements and laboratory investigation,Complication monitor sheet Pre-implantation of clinical pathway.

\section{2- Implementation phase:}

The researcher implement the clinical pathway step by step for the oncology staff consultant and nurse, Pharmacist, technician those responsible for providing clinical pathway.

The steps of intervention guidelines implementation.

The researcher will be available in the study settings four days per week consecutive, at the mooring shift from 8.00 A.M to 2.00 P.M in Tanta Cancer Center Affiliated to the Ministry of Health (Pediatric Oncology Department) and Out Patient Clinic of Oncology and Inpatient Pediatric Oncology Department in Benha Children Hospital.

The researcher explained the aim of the study and asked for the hospital team for cooperation.

Children with cancer suffering from neutropenia will be divided into two equal groups (study and control group).

The implementation of clinical pathway will be applied to all children in the study group and divided into 5 sessions. Each session will be expected to take duration of 
30 minutes and will be given to each individually with family member

Discussion and power point for illustration

will be used for each child individually.

The first session: It was focus on assess the children undergoing chemotherapy with neutropenia, assess all hematological system for study and control group .

The second session: It was concentrated on select the children which having neutropenia, assess all hematological system for study and control group.

The third session: Document all gathered data for comparison to evaluate the efficacy of clinical pathway.

The fourth session: Application of clinical pathway for study group only by all nursing staff included the following items :Feeding habit and problems, daily living activity, nutritional assessment sheet of cancer child Physical assessment sheet of cancer child .Anthropometric measurement .Laboratory investigations

Daily dietary intake

The fifth session :Safety practice during medication administration: that was included nine right, dose calculation, continuous monitoring and observation of side effect. - Medications intake which include the following.Blood transfusion (taken, not taken).Platelet intake (taken, not taken).Antibiotic intravenous (oral, single, double).Antipyretic (taken, not
taken).Chemotherapy (taken, not taken).Growth factors (taken, not taken)Steroids: (taken, not taken).

\section{3- Evaluation phase:}

Evaluate the effect of clinical pathway outcome on studied group and compare them with control group who received routine care by using the four tools three times pretest, immediate after clinical pathway implementation and follow up one month.

\section{4-Statistical analysis:}

An IBM compatible PC was used to store and analyze the data. Calculations were done by means of the Statistical Package for Social Sciences SPSS version 17. Data colleted were revised, coded and analyzed, and presented using descriptive statistics in the form of frequencies and percentages for qualitative variables; Means, standard deviations and $\mathrm{z}$-score, for quantitative data. Test of significance was used for comparison between the study and control groups.Where ,P > 0.05 No statistically significant difference. $\mathrm{P}<0.05^{*}$ statistically significant difference.P < $0.001^{* *}$ highly statistically significant difference.

\section{Results}

Table (1) :Regarding to age, it was observed that nearly more than half of control group $\&$ half of the studied group age ranged between 8:10 year with mean 


\section{Tanta Scientific Nursing Journal}

\pm SD $9.75 \pm 4.86,9.38 \pm 4.39$ respectively. It was found that $61.1 \%, 50.5 \%$ were male in the studied and control group respectively. Regarding to educational level it was found that majority (94.4\%) of the studied $\&$ more than three quarter $(77.8 \%)$ of the control group had primary school level respectively .

Table (2): percentage distribution of studied children in relation to diagnosis it was found that two third $(63.9 \%, 72.2 \%)$ of the studied \& control group suffering from leukemia .Regarding to time of hospital stay more than half $63.9 \%$ of the studied $\&$ most $(86.1 \%)$ of the control group stay more than 10 days . in relation to school attendance it was found that $(77.8 \%, 66.7 \%$ )of the studied \& control group not attended to school in relation to cause of absenteeism it was observed that more than half of the studed(85.3\%)\&nearly two third (66.7\% )of the control group absent due to hospitalization. Their was a negative family history to cancer in children presented by ( $80.6 \%$ )of studied and control group respectively.

Table (4): Illustrates percentage distribution of studied children in relation to dietary profile, it was observed that in first five days, their were no statistically significant differences between the control and study group regarding to, feeding habits \& problem including, feeding problem which, nausea, vomiting, loss of appitite, dysphagia and dry mouth were $\left(\mathrm{p}=0.003^{* *}, \quad \mathrm{P}=0.380, \quad \mathrm{P}=0.235, \mathrm{P}=1.0\right.$, $\mathrm{P}=0.898, \mathrm{P}=0.495, \mathrm{P}=0.722)$ respectively. At second five days and third five days, it was observed that their were a highly statistically significant differences in the studied children between two groups were $\left(\mathrm{p}=0.001^{* *}\right)$ respectively .

Table (5): Illustrate percentage distribution of studied children suffering from neutropenia under chemotherapy related to daily activity, activity and physical examination. It was found that at first five days, there were no statistically significant differences between the study and control group regarding to, daily activity, level of activity and physical assessment of children which included, hair, face, lips, gums, gastrointestinal tract and skeletal system were $(\mathrm{P}=0.197$, $\mathrm{P}=0.234, \quad \mathrm{P}=0.453, \quad \mathrm{P}=0.074$, $\mathrm{P}=0.088, \mathrm{P}=0.496, \quad \mathrm{P}=0.144, \quad \mathrm{P}=0.058)$ respectively. At second five and third five days, there were a highly statistically significant differences in the studied and control group were $(\mathrm{p}=0.001 * *)$ respectively

Table (6): Illustrate percentage distribution of studied children suffering from neutropenia under chemotherapy related to vital signs, it was found that at first five days, there was a statistically 


\section{Tanta Scientific Nursing Journal}

significant differences between the study and control group regarding to body temperature $\left(\mathrm{p}=0.006^{* *}\right)$, but there were no a statistically significant differences between the control and study group regarding to pulse, respiration and blood pressure $\quad(\mathrm{p}=1.0, \mathrm{p}=0.751, \mathrm{p}=0.892)$ respectively. At second \&third five days, there were a highly statistically significant differences between study and control group $\left(\mathrm{p}=0.001^{* *}\right)$ respectively .

Table (7): Illustrate percentage distribution of studied children suffering from neutropenia under chemotherapy related to anthropometric measurements, it was found that at first five days, their was no a statistically significant differences between the study and control group regarding to weight, hight, arm circumference, subceatuous fat, body surface area $(\mathrm{p}=0.807, \mathrm{P}=0.075, \mathrm{P}=0.453$, $\mathrm{P}=0.394, \mathrm{P}=0.059)$ respectively. At second and third five days, their were a highly statistically significant differences in the studied children between two groups related to weight and body surface area were $(\mathrm{p}=0.002 * *, \mathrm{p}=0.001 * *)$ respectively. Their was a statistically significant differences between study and control group in relation to height at second five days $\left(\mathrm{p}=0.013^{*}\right)$. And their was no statistically significant differences in the studied children between between study and control group related to arm circumference at second and third five days were $(\mathrm{p}=0.088, \mathrm{p}=0.077)$ respectively.

Table (8): Illustrate percentage distribution of studied children suffering from neutropenia under chemotherapy $r$ related to laboratory investigation, it was found that at first five days, their were no a statistically significant differences between between study and control group regarding to complete blood count, lactic acid dehydrogenises, renal function test and bone marrow aspiration were $(\mathrm{p}=1.0$, $\mathrm{p}=0.727, \mathrm{p}=0.059, \mathrm{p}=0.551)$ respectively. At second and third five days, their were a highly statistically significant differences in the studied children between study and control group related to lactic acid dehydrogenises, renal function test and bone marrow aspiration were $(\mathrm{P}=0.001 * *$, $\left.\mathrm{P}=0.005^{* *}\right)$ respectively .

Table (9): Illustrate percentage distribution of studied children suffering from neutropenia under chemotherapy $r$ related to general complication, it was found that at first five days, their were no a statistically significant differences between the study and control group regarding to low platelet count, loss of appetite, loss of weight and hyperthermia were $(\mathrm{P}=1.0$, $\mathrm{P}=0.285, \mathrm{P}=0.280, \mathrm{P}=0.058)$ respectively. At second and third five days, their were a highly statistically significant differences 


\section{Tanta Scientific Nursing Journal}

in the studied children between study and control group related to all general complication were $(\mathrm{P}=0.001 * *)$.

Table (10): Illustrate percentage distribution of studied children suffering from neutropenia under chemotherapy related to specific complication, it was found that at first five days, their were no a statistically significant differences between the study and control group regarding to specific complication which included, inflammation of mucous membrane, diarrhea, loss of hair, neurological system, level of activity, muscular and respiratory system, were $(\mathrm{p}=0.134, \mathrm{p}=0.173, \mathrm{p}=0.633$, $\mathrm{p}=0.437, \quad \mathrm{p}=0.182, \mathrm{p}=0.173, \mathrm{p}=0.101$, $\mathrm{p}=0.722$ respectively. At second and third five days, their were a highly statistically significant differences in the studied children between two groups related to all specific complication were $(\mathrm{p}=0.001 * *)$ 
Table (1): Percentage distribution of studied children suffering from neutropenia under chemotherapy related to Biosociodemographic characteristics.

\begin{tabular}{|c|c|c|c|c|}
\hline \multirow{3}{*}{$\begin{array}{c}\text { Biosocio } \\
\text { Demographic } \\
\text { Characteristics }\end{array}$} & \multicolumn{2}{|c|}{$(n=36)$} & \multicolumn{2}{|c|}{$(n=36)$} \\
\hline & \multicolumn{2}{|c|}{ Study group } & \multicolumn{2}{|c|}{ Control group } \\
\hline & No & $\%$ & No & $\%$ \\
\hline \multicolumn{5}{|l|}{ Age in years } \\
\hline $\begin{array}{l}6>8< \\
8>10 \\
10-12\end{array}$ & $\begin{array}{c}8 \\
25 \\
3\end{array}$ & $\begin{array}{c}22.2 \\
69.4 \\
8.3 \\
\end{array}$ & $\begin{array}{c}14 \\
18 \\
4\end{array}$ & $\begin{array}{l}38.9 \\
50.0 \\
11.1\end{array}$ \\
\hline Mean \pm SD & \multicolumn{2}{|c|}{$9.75 \pm 4.86$} & \multicolumn{2}{|c|}{$9.38 \pm 4.39$} \\
\hline $\begin{array}{c}\text { Sex } \\
\text { Male } \\
\text { Female }\end{array}$ & $\begin{array}{l}22 \\
14\end{array}$ & $\begin{array}{l}61.1 \\
38.9\end{array}$ & $\begin{array}{l}18 \\
18\end{array}$ & $\begin{array}{l}50.0 \\
50.0\end{array}$ \\
\hline $\begin{array}{l}\text { Educational level } \\
\text { Primary school } \\
\text { Preparatory school }\end{array}$ & $\begin{array}{c}34 \\
2\end{array}$ & $\begin{array}{c}94.4 \\
5.6\end{array}$ & $\begin{array}{c}28 \\
8\end{array}$ & $\begin{array}{l}77.8 \\
22.2\end{array}$ \\
\hline
\end{tabular}


Table (2):Percentage distribution of studied children suffering from neutropenia under chemotherapy related to medical history.

\begin{tabular}{|c|c|c|c|c|c|c|}
\hline & \multirow{2}{*}{\multicolumn{2}{|c|}{$\begin{array}{l}(\mathrm{n}=36) \\
\begin{array}{l}\text { Study } \\
\text { group }\end{array}\end{array}$}} & \multirow{2}{*}{\multicolumn{2}{|c|}{$\begin{array}{c}(\mathrm{n}=36) \\
\begin{array}{c}\text { Control } \\
\text { group }\end{array} \\
\end{array}$}} & \multirow{2}{*}{\multicolumn{2}{|c|}{$\begin{array}{l}(\mathrm{n}=72) \\
\text { Total }\end{array}$}} \\
\hline & & & & & & \\
\hline & No & $\%$ & No & $\%$ & No & $\%$ \\
\hline Diagnosis & \multirow[b]{2}{*}{$\begin{array}{l}23 \\
13 \\
\end{array}$} & \multirow[b]{2}{*}{$\begin{array}{l}63.9 \\
36.1 \\
\end{array}$} & \multirow[b]{2}{*}{$\begin{array}{l}26 \\
10 \\
\end{array}$} & \multirow[b]{2}{*}{$\begin{array}{l}72.2 \\
27.8 \\
\end{array}$} & \multirow[b]{2}{*}{$\begin{array}{l}49 \\
23 \\
\end{array}$} & \multirow[b]{2}{*}{$\begin{array}{l}68.1 \\
31.9 \\
\end{array}$} \\
\hline $\begin{array}{l}\text { Leukemia } \\
\text { sold tumors }\end{array}$ & & & & & & \\
\hline \multicolumn{7}{|l|}{ Time of hospital stay in days } \\
\hline $\begin{array}{c}1-5 \\
>5 \\
>10 \text { days } \\
\end{array}$ & $\begin{array}{c}2 \\
11 \\
23 \\
\end{array}$ & $\begin{array}{l}5.6 \\
30.6 \\
63.9 \\
\end{array}$ & $\begin{array}{c}0 \\
5 \\
31 \\
\end{array}$ & $\begin{array}{l}.0 \\
13.9 \\
86.1 \\
\end{array}$ & $\begin{array}{c}2 \\
16 \\
54 \\
\end{array}$ & $\begin{array}{c}2.8 \\
22.2 \\
75.0\end{array}$ \\
\hline \multicolumn{7}{|l|}{ Times of hospital admission } \\
\hline $\begin{array}{l}\text { Once } \\
\text { Twice more than twice } \\
\text { Others } \\
\end{array}$ & $\begin{array}{c}1 \\
18 \\
17 \\
\end{array}$ & $\begin{array}{l}2.8 \\
50.0 \\
47.2 \\
\end{array}$ & $\begin{array}{c}3 \\
12 \\
21 \\
\end{array}$ & $\begin{array}{l}8.3 \\
33.3 \\
58.3 \\
\end{array}$ & $\begin{array}{c}4 \\
30 \\
38 \\
\end{array}$ & $\begin{array}{l}5.6 \\
41.7 \\
52.8 \\
\end{array}$ \\
\hline \multicolumn{7}{|l|}{ School attendance } \\
\hline $\begin{array}{l}\text { Yes } \\
\text { No }\end{array}$ & $\begin{array}{c}8 \\
28\end{array}$ & $\begin{array}{l}22.2 \\
77.8\end{array}$ & $\begin{array}{l}12 \\
24\end{array}$ & $\begin{array}{l}33.3 \\
66.7\end{array}$ & $\begin{array}{l}20 \\
52\end{array}$ & $\begin{array}{l}27.8 \\
72.2\end{array}$ \\
\hline \multicolumn{7}{|l|}{ Causes } \\
\hline $\begin{array}{l}\text { bad psychological status } \\
\text { hospitalization } \\
\text { general fatigue } \\
\text { other }\end{array}$ & $\begin{array}{c}4 \\
21 \\
0 \\
11 \\
\end{array}$ & $\begin{array}{l}11.1 \\
58.3 \\
.0 \\
30.6 \\
\end{array}$ & $\begin{array}{c}3 \\
24 \\
1 \\
7 \\
\end{array}$ & $\begin{array}{l}8.3 \\
66.7 \\
2.8 \\
19.4 \\
\end{array}$ & $\begin{array}{c}7 \\
45 \\
1 \\
18 \\
\end{array}$ & $\begin{array}{l}9.7 \\
62.5 \\
1.4 \\
25.0 \\
\end{array}$ \\
\hline \multicolumn{7}{|l|}{ Hereditaryfactors } \\
\hline $\begin{array}{l}\text { Yes } \\
\text { No }\end{array}$ & $\begin{array}{c}7 \\
29\end{array}$ & $\begin{array}{l}14.9 \\
80.6\end{array}$ & $\begin{array}{l}21 \\
15\end{array}$ & $\begin{array}{l}19.4 \\
80.6\end{array}$ & $\begin{array}{l}38.9 \\
61.1\end{array}$ & $\begin{array}{l}29.2 \\
70.8\end{array}$ \\
\hline
\end{tabular}


Tanta Scientific Nursing Journal

Table (3): Percentage distribution of studied children suffering from neutropenia under chemotherapy related to dietary profile

\begin{tabular}{|c|c|c|c|c|c|c|c|c|c|c|c|c|c|c|c|c|c|c|c|}
\hline & & \multicolumn{4}{|c|}{$\begin{array}{c}\begin{array}{c}\text { First five days } \\
\text { no }=72\end{array} \\
\end{array}$} & \multicolumn{2}{|c|}{ Chi square } & \multicolumn{4}{|c|}{$\begin{array}{c}\begin{array}{c}\text { Second five days } \\
\text { no=72 }\end{array} \\
\end{array}$} & \multicolumn{2}{|c|}{ Chi square } & \multicolumn{4}{|c|}{$\begin{array}{c}\text { Third five days } \\
\text { no=72 }\end{array}$} & \multicolumn{2}{|c|}{ Chi square } \\
\hline & & \multicolumn{2}{|c|}{ Study group } & \multicolumn{2}{|c|}{$\begin{array}{c}\text { Control } \\
\text { group }\end{array}$} & \multirow[t]{2}{*}{${ }^{2} \mathbf{X}$} & \multirow{2}{*}{$\begin{array}{c}\mathbf{P} \\
\text { value }\end{array}$} & \multicolumn{2}{|c|}{$\begin{array}{l}\text { Study } \\
\text { group }\end{array}$} & \multicolumn{2}{|c|}{$\begin{array}{l}\text { Control } \\
\text { group }\end{array}$} & \multirow[t]{2}{*}{$\mathbf{X}^{2}$} & \multirow[t]{2}{*}{$P$ value } & \multicolumn{2}{|c|}{ Study group } & \multicolumn{2}{|c|}{$\begin{array}{c}\begin{array}{c}\text { Control } \\
\text { group }\end{array} \\
\end{array}$} & \multirow[t]{2}{*}{$\mathbf{X}^{2}$} & \multirow[t]{2}{*}{$P$ value } \\
\hline & & No & $\%$ & No & $\%$ & & & No & $\%$ & No & $\%$ & & & No & $\%$ & No & $\%$ & & \\
\hline \multicolumn{18}{|l|}{ Dietary profile } & \multirow{4}{*}{49.04} & \multirow{4}{*}{$0.001 * *$} \\
\hline \begin{tabular}{|c|}
$\begin{array}{c}\text { Number of meal } \\
\text { per day }\end{array}$ \\
\end{tabular} & 1 & 34 & 94.4 & 19 & 52.8 & \multirow[t]{3}{*}{16.38} & \multirow{3}{*}{$\begin{array}{c}0.003 * \\
*\end{array}$} & 0 & .0 & 25 & 69.4 & \multirow[t]{3}{*}{38.30} & \multirow[t]{3}{*}{$0.001 * *$} & 0 & .0 & 24 & 66.7 & & \\
\hline & 2 & 2 & 5.6 & 12 & 33.3 & & & 33 & 91.7 & 10 & 27.8 & & & 11 & 30.6 & 12 & 33.3 & & \\
\hline & 3 & 0 & .0 & 5 & 13.9 & & & 3 & 8.3 & 1 & 2.8 & & & 25 & 69.4 & 0 & .0 & & \\
\hline \multirow[t]{3}{*}{ Appetite } & & & & & & \multirow{3}{*}{1.93} & \multirow{3}{*}{0.380} & & & & & \multirow{3}{*}{47.04} & & & & & & & \\
\hline & Like & 1 & 2.8 & 4 & 11.1 & & & 31 & 86.1 & 2 & 5.6 & & $0.001 * *$ & 36 & 100.0 & 0 & .0 & 72.00 & $0.001 * *$ \\
\hline & Dislike & 35 & 97.2 & 32 & 88.9 & & & 5 & 13.9 & 34 & 94.4 & & & 0 & .0 & 36 & 100.0 & & \\
\hline \begin{tabular}{|l|} 
Feeding problem \\
\end{tabular} & & & & & & & & & & & & & & & & & & & \\
\hline Nausea & Yes & 31 & 86.1 & 25 & 69.4 & 2.89 & 0.235 & 5 & 13.9 & 34 & 94.4 & 47.04 & $0.001 * *$ & 0 & .0 & 32 & 88.9 & 57.60 & $0.001 * *$ \\
\hline & No & 5 & 13.9 & 11 & 30.6 & & & 31 & 86.1 & 2 & 5.6 & & & 36 & 100.0 & 4 & 11.1 & & \\
\hline Vomiting & Yes & 33 & 91.7 & 33 & 91.7 & & & 4 & 11.1 & 35 & 97.2 & & & 0 & .0 & 29 & 80.6 & & \\
\hline & No & 3 & 8.3 & 3 & 8.3 & 0.000 & 1.0 & 32 & 88.9 & 1 & 2.8 & 53.10 & 0.001 & 36 & 100.0 & 7 & 19.4 & 48.55 & $0.001 \cdots$ \\
\hline \begin{tabular}{|l|} 
Loss of appetite \\
\end{tabular} & Yes & 33 & 91.7 & 34 & 94.4 & & & 8 & 22.2 & 34 & 94.4 & & & 0 & .0 & 31 & 86.1 & & \\
\hline & No & 3 & 8.3 & 2 & 5.6 & 0.215 & 0.898 & 28 & 77.8 & 2 & 5.6 & 38.02 & $0.001 \times$ & 36 & 100.0 & 5 & 13.9 & 54.43 & $0.001 \times$ \\
\hline Dysphgia & Yes & 32 & 88.9 & 30 & 83.3 & & & 5 & 13.9 & 33 & 91.7 & & & 0 & .0 & 32 & 88.9 & & \\
\hline & No & 4 & 11.1 & 6 & 16.7 & 0.405 & 0.495 & 31 & 86.1 & 3 & 8.3 & 43.09 & 0.001 & 36 & 100.0 & 4 & 11.1 & $5 \% .00$ & $0.001 \%$ \\
\hline Dryness mouth & Yes & 31 & 86.1 & 32 & 88.9 & 0127 & 0722 & 4 & 11.1 & 35 & 97.2 & 53.76 & & 0 & .0 & 35 & 97.2 & 68.10 & \\
\hline & No & 5 & 13.9 & 4 & 11.1 & 0.121 & 0.122 & 32 & 88.9 & 1 & 2.8 & 53.10 & 0.001 & 36 & 100.0 & 1 & 2.8 & 08.10 & 0.001 \\
\hline
\end{tabular}

At first five days: no significant

At second five days: highly significant at level $p<0.001 * *$

At third five days: High significant at level $p<0.001 * *$

Vol. 12 No. 1 May, 2017 


\section{Tanta Scientific Nursing Journal}

Table (4): Percentage distribution of studied children suffering from neutropenia under chemotherapy related to daily activity, activity and physical examination.

\begin{tabular}{|c|c|c|c|c|c|c|c|c|c|c|c|c|c|c|c|c|c|c|c|}
\hline & & \multicolumn{4}{|c|}{$\begin{array}{c}\begin{array}{c}\text { First five days } \\
\text { no=72 }\end{array} \\
\end{array}$} & \multicolumn{2}{|c|}{ Chi square } & \multicolumn{4}{|c|}{$\begin{array}{c}\begin{array}{c}\text { Second five days } \\
\text { no=72 }\end{array} \\
\end{array}$} & \multicolumn{2}{|c|}{ Chi square } & \multicolumn{4}{|c|}{$\begin{array}{c}\begin{array}{c}\text { Third five days } \\
\text { no=72 }\end{array} \\
\end{array}$} & \multicolumn{2}{|c|}{ Chi square } \\
\hline & & \multicolumn{2}{|c|}{ Study group } & \multicolumn{2}{|c|}{$\begin{array}{c}\text { Control } \\
\text { group }\end{array}$} & \multirow{2}{*}{$\mathrm{X}^{2}$} & \multirow{2}{*}{$P$ value } & \multicolumn{2}{|c|}{ Study group } & \multicolumn{2}{|c|}{ Control group } & \multirow{2}{*}{$\mathrm{X}^{2}$} & \multirow{2}{*}{$P$ value } & \multicolumn{2}{|c|}{ Study group } & \multicolumn{2}{|c|}{ Control group } & \multirow{2}{*}{$\mathrm{X}^{2}$} & \multirow{2}{*}{$P$ value } \\
\hline & & No & $\%$ & No & $\%$ & & & no & $\%$ & No & $\%$ & & & No & $\%$ & No & $\%$ & & \\
\hline \multirow{4}{*}{ Daily activity } & & & & & & \multirow{4}{*}{3.37} & \multirow{4}{*}{0.197} & & & & & \multirow{4}{*}{44.50} & \multirow{4}{*}{$0.001 * *$} & & & & & \multirow{4}{*}{52.72} & \multirow{4}{*}{$0.001 * *$} \\
\hline & hyper activity & 0 & .0 & 3 & 8.3 & & & 6 & 16.7 & 2 & 5.6 & & & 30 & 83.3 & 0 & .0 & & \\
\hline & Normal & 4 & 11.1 & 5 & 13.9 & & & 28 & 77.8 & 4 & 11.1 & & & 3 & 8.3 & 7 & 19.4 & & \\
\hline & limited activity & 32 & 88.9 & 28 & 77.8 & & & 2 & 5.6 & 30 & 83.3 & & & 3 & 8.3 & 29 & 80.6 & & \\
\hline \multirow{4}{*}{ Level of activity } & no play & 31 & 86.1 & 27 & 75.0 & \multirow{4}{*}{1.41} & & 0 & .0 & 24 & 66.7 & & & 0 & .0 & 23 & 63.9 & & \\
\hline & Quite play & 5 & 13.9 & 9 & 25.0 & & 0234 & 32 & 88.9 & 11 & 30.6 & 3605 & $0001 * *$ & 2 & 5.6 & 12 & 33.3 & 6126 & $0001 *$ \\
\hline & active play & 0 & .0 & 0 & .0 & & 0.234 & 4 & 11.1 & 1 & 2.8 & 30.05 & $0.0011^{2}$ & 32 & 88.9 & 1 & 2.8 & 01.20 & $0.001^{*}$ \\
\hline & group play & 0 & .0 & 0 & .0 & & & 0 & .0 & 0 & .0 & & & 2 & 5.6 & 0 & .0 & & \\
\hline physical & Yes & 31 & 86.1 & 33 & 91.7 & & & 31 & 86.1 & 35 & 97.2 & & & 32 & 88.9 & 32 & 88.9 & & \\
\hline $\begin{array}{l}\text { assessment for } \\
\text { children hair }\end{array}$ & No & 5 & 13.9 & 3 & 8.3 & 0.563 & 0.453 & 5 & 13.9 & 1 & 2.8 & 2.90 & 0.088 & 4 & 11.1 & 4 & 11.1 & 0.00 & 1.0 \\
\hline Face & Normal & 4 & 11.1 & 10 & 27.8 & 310 & 0074 & 32 & 88.9 & 6 & 16.7 & 3767 & $0001 * *$ & 34 & 94.4 & 7 & 19.4 & 4120 & $0001 * *$ \\
\hline Face & Abnormal & 32 & 88.9 & 26 & 72.2 & 3.19 & $0.0 / 4$ & 4 & 11.1 & 30 & 83.3 & 31.6\% & $0.001 \%$ & 2 & 5.6 & 29 & 80.6 & 41.29 & $0.001{ }^{*}$ \\
\hline Fye & Normal & 2 & 5.6 & 8 & 22.2 & 418 & $0041 *$ & 29 & 80.6 & 4 & 11.1 & 3496 & $0001 * *$ & 36 & 100.0 & 10 & 27.8 & 4060 & $0001 * *$ \\
\hline Буе & Abnormal & 34 & 94.4 & 28 & 77.8 & 4.10 & $0.041^{2}$ & 7 & 19.4 & 32 & 88.9 & 34.90 & 0.001 & 0 & .0 & 26 & 72.2 & 4.09 & 0.001 \\
\hline Lins & Normal & 1 & 2.8 & 5 & 13.9 & 200 & 0088 & 30 & 83.3 & 2 & 5.6 & & & 36 & 100.0 & 0 & .0 & & \\
\hline Lips & Abnormal & 35 & 97.2 & 31 & 86.1 & 2.90 & 0.088 & 6 & 16.7 & 34 & 94.4 & 44.10 & $0.001^{* * *}$ & 0 & .0 & 36 & 100.0 & 72.00 & $0.001^{* *}$ \\
\hline Gums & Normal & 4 & 11.1 & 6 & 16.7 & 0.462 & 0.496 & 34 & 94.4 & 2 & 5.6 & 56.88 & $0.001 * *$ & 36 & 100.0 & 2 & 5.6 & 64.42 & $0.001 * *$ \\
\hline Gums & Abnormal & 32 & 88.9 & 30 & 83.3 & 0.462 & 0.496 & 2 & 5.6 & 34 & 94.4 & 56.88 & $0.001 \%$ & 0 & .0 & 34 & 94.4 & 64.42 & $0.001{ }^{2}=$ \\
\hline GIT & Normal & 7 & 19.4 & 13 & 36.1 & 2.49 & 0.114 & 29 & 80.6 & 8 & 22.2 & 24.51 & $0.001 * *$ & 35 & 97.2 & 7 & 19.4 & 44.80 & $0.001 * *$ \\
\hline Gil & Abnormal & 29 & 80.6 & 23 & 63.9 & 2.49 & 0.114 & 7 & 19.4 & 28 & 77.8 & 24.51 & $0.001 \%$ & 1 & 2.8 & 29 & 80.6 & 44.80 & $0.001{ }^{2}=$ \\
\hline Steletols & Normal & 27 & $75.0 \%$ & 33 & $91.7 \%$ & 360 & 0058 & 11 & 30.6 & 30 & 83.3 & 20.45 & * & 3 & 8.3 & 30 & 83.3 & 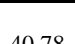 & $0001 * *$ \\
\hline Skeletal system & Abnormal & 9 & $25.0 \%$ & 3 & $8.3 \%$ & 3.00 & 0.038 & 25 & 69.4 & 6 & 16.7 & 20.45 & 0.001 & 33 & 91.7 & 6 & 16.7 & 40.18 & $0.001 \%$ \\
\hline
\end{tabular}

Significant at level $p<0.001 * *$ 
Tanta Scientific Nursing Journal

Table (5): Percentage distribution of studied children suffering from neutropenia under chemotherapy related to vital signs

\begin{tabular}{|c|c|c|c|c|c|c|c|c|c|c|c|c|c|c|c|c|c|c|c|}
\hline & & \multicolumn{4}{|c|}{$\begin{array}{c}\text { First five days } \\
\text { no=72 }\end{array}$} & \multicolumn{2}{|c|}{ Chi square } & \multicolumn{4}{|c|}{$\begin{array}{c}\text { Second five days } \\
\text { no=72 }\end{array}$} & \multicolumn{2}{|c|}{ Chi square } & \multicolumn{4}{|c|}{$\begin{array}{c}\text { Third five days } \\
\text { no=72 }\end{array}$} & \multicolumn{2}{|c|}{ Chi square } \\
\hline & & \multicolumn{2}{|c|}{$\begin{array}{l}\text { Study } \\
\text { group }\end{array}$} & \multicolumn{2}{|c|}{$\begin{array}{l}\text { Control } \\
\text { group }\end{array}$} & \multirow{2}{*}{$X^{2}$} & \multirow{2}{*}{$P$ value } & \multicolumn{2}{|c|}{$\begin{array}{l}\text { Study } \\
\text { group }\end{array}$} & \multicolumn{2}{|c|}{$\begin{array}{l}\text { Control } \\
\text { group }\end{array}$} & \multirow{2}{*}{$\mathbf{X} 2$} & \multirow{2}{*}{$P$ value } & \multicolumn{2}{|c|}{ Study group } & \multicolumn{2}{|c|}{ Control group } & \multirow{2}{*}{$\mathrm{X} 2$} & \multirow{2}{*}{$P$ value } \\
\hline & & No & $\%$ & No & $\%$ & & & No & $\%$ & No & $\%$ & & & No & $\%$ & No & $\%$ & & \\
\hline \multirow[t]{2}{*}{ Temperature } & Normal & 0 & .0 & 6 & 16.7 & \multirow[t]{2}{*}{10.28} & \multirow[t]{2}{*}{$0.006^{* *}$} & 30 & 83.3 & 2 & 5.6 & \multirow[t]{2}{*}{47.55} & \multirow[t]{2}{*}{$0.001 * *$} & 36 & 100.0 & 9 & 25.0 & \multirow[t]{2}{*}{43.20} & \multirow[t]{2}{*}{$0.001 * *$} \\
\hline & Abnormal & 36 & 100.0 & 27 & 75.0 & & & 3 & 8.3 & 31 & 86.1 & & & 0 & .0 & 20 & 55.6 & & \\
\hline \multirow{2}{*}{ Pulse } & Normal & 0 & 0 & 0 & 0 & \multirow{3}{*}{0.00} & \multirow{3}{*}{1.0} & 0 & 0 & 0 & 0 & \multirow{3}{*}{50.20} & \multirow{3}{*}{$0.001 * *$} & 0 & 0 & 0 & 0 & \multirow{3}{*}{29.64} & \multirow{3}{*}{$0.001 * *$} \\
\hline & Abnormal & 2 & 5.6 & 2 & 5.6 & & & 32 & 88.9 & 2 & 5.6 & & & 36 & 100.0 & 15 & 41.7 & & \\
\hline \multirow{2}{*}{ Respiration } & Normal & 0 & 0 & 0 & 0 & & & 0 & .0 & 1 & 2.8 & & & 0 & .0 & 1 & 2.8 & & \\
\hline & Abnormal & 27 & 75.0 & 29 & 80.6 & 0.321 & 0.571 & 35 & 97.2 & 15 & 41.7 & 26.18 & $0.001 * *$ & 36 & 100.0 & 32 & 88.9 & 4.23 & $0.039 *$ \\
\hline \multirow{2}{*}{$\begin{array}{c}\text { Blood } \\
\text { pressure }\end{array}$} & Normal & 8 & 22.2 & 7 & 19.4 & חרת 0 & 0 & 34 & 94.4 & 11 & 30.6 & 50 & , & 36 & 100.0 & 13 & 36.1 & 70 & $1 \%$. \\
\hline & Abnormal & 20 & 55.6 & 22 & 61.1 & & 0.072 & 2 & 5.6 & 19 & 52.8 & ס & 0.001 & 0 & .0 & 18 & 50.0 & אור. & 0.001 \\
\hline
\end{tabular}

Significant at level $\mathbf{p}<0.001 * *$

Vol. 12 No. 1 May, 2017 
Table (6): Percentage distribution of studied children suffering from neutropeniaunder chemotherapy related to anthropometric measurements.

\begin{tabular}{|c|c|c|c|c|c|c|c|c|c|c|c|c|c|c|c|c|c|c|c|}
\hline & & \multicolumn{4}{|c|}{ First five days no=72 } & \multicolumn{2}{|c|}{ Chi square } & \multicolumn{4}{|c|}{ Second five days $\quad$ no $=72$} & \multicolumn{2}{|c|}{ Chi square } & \multicolumn{4}{|c|}{$\begin{array}{c}\text { Third five days } \\
\text { no }=72\end{array}$} & \multicolumn{2}{|c|}{ Chi square } \\
\hline & & \multicolumn{2}{|c|}{$\begin{array}{l}\text { Study } \\
\text { group }\end{array}$} & \multicolumn{2}{|c|}{$\begin{array}{c}\begin{array}{c}\text { Control } \\
\text { group }\end{array} \\
\end{array}$} & \multirow{2}{*}{$\mathbf{X}^{2}$} & \multirow{2}{*}{$\begin{array}{c}P \\
\text { value }\end{array}$} & \multicolumn{2}{|c|}{$\begin{array}{l}\text { Study } \\
\text { Group }\end{array}$} & \multicolumn{2}{|c|}{ Control group } & \multirow[t]{2}{*}{$\mathbf{X}^{2}$} & \multirow[t]{2}{*}{$P$ value } & \multicolumn{2}{|c|}{$\begin{array}{l}\text { Study } \\
\text { group }\end{array}$} & \multicolumn{2}{|c|}{$\begin{array}{c}\text { Control } \\
\text { group }\end{array}$} & \multirow[t]{2}{*}{$\mathbf{X}^{2}$} & \multirow{2}{*}{$\begin{array}{c}P \\
\text { value }\end{array}$} \\
\hline & & No & $\%$ & No & $\%$ & & & No & $\%$ & No & $\%$ & & & No & $\%$ & No & $\%$ & & \\
\hline \multirow[b]{2}{*}{ Weight } & Normal & 13 & 36.1 & 14 & 38.9 & \multirow[b]{2}{*}{0.059} & \multirow[b]{2}{*}{0.807} & 27 & 75.0 & 14 & 38.9 & \multirow[b]{2}{*}{9.57} & \multirow[b]{2}{*}{$0.002^{* *}$} & 33 & 91.7 & 13 & 36.1 & \multirow[b]{2}{*}{24.08} & \multirow[b]{2}{*}{$\begin{array}{c}0.001 * \\
*\end{array}$} \\
\hline & $\begin{array}{c}\text { Abnorma } \\
1\end{array}$ & 23 & 63.9 & 22 & 61.1 & & & 9 & 25.0 & 22 & 61.1 & & & 3 & 8.3 & 23 & 63.9 & & \\
\hline \multirow[b]{2}{*}{ hight } & Normal & 34 & 94.4 & 29 & 80.6 & \multirow[b]{2}{*}{3.17} & \multirow[b]{2}{*}{0.075} & 35 & 97.2 & 28 & 77.8 & \multirow[b]{2}{*}{6.22} & \multirow[b]{2}{*}{$0.013^{*}$} & 36 & 100.0 & 28 & 77.8 & \multirow[b]{2}{*}{9.00} & \multirow[b]{2}{*}{$\begin{array}{c}0.003 * \\
*\end{array}$} \\
\hline & $\begin{array}{c}\text { Abnorma } \\
1\end{array}$ & 2 & 5.6 & 7 & 19.4 & & & 1 & 2.8 & 8 & 22.2 & & & 0 & .0 & 8 & 22.2 & & \\
\hline \multirow[b]{2}{*}{$\begin{array}{c}\text { Mid Arm } \\
\text { circumference }\end{array}$} & Normal & 33 & 91.7 & 31 & 86.1 & & & 35 & 97.2 & 31 & 86.1 & & & 36 & 100.0 & 33 & 91.7 & & \\
\hline & $\begin{array}{c}\text { Abnorma } \\
1\end{array}$ & 3 & 8.3 & 5 & 13.9 & 0.563 & 0.453 & 1 & 2.8 & 5 & 13.9 & 2.90 & 0.088 & 0 & .0 & 3 & 8.3 & 3.13 & 0.077 \\
\hline & Normal & 34 & 94.4 & 32 & 88.9 & & & 36 & 100.0 & 33 & 91.7 & & & 36 & 100.0 & 19 & 52.8 & & \\
\hline $\begin{array}{c}\text { Subceatuous } \\
\text { fat }\end{array}$ & $\begin{array}{c}\text { Abnorma } \\
1\end{array}$ & 2 & 5.6 & 4 & 11.1 & 0.727 & 0.394 & 0 & .0 & 3 & 8.3 & 3.13 & 0.077 & 0 & .0 & 17 & 47.2 & 22.25 & $\begin{array}{c}0.001 * \\
*\end{array}$ \\
\hline Body mase & Normal & 22 & 61.1 & 14 & 38.9 & & & 32 & 88.9 & 17 & 47.2 & & & 32 & 88.9 & 17 & 47.2 & & \\
\hline index & $\begin{array}{c}\text { Abnorma } \\
1\end{array}$ & 14 & 38.9 & 22 & 61.1 & 3.55 & 0.059 & 4 & 11.1 & 19 & 52.8 & 14.37 & $0.001 * *$ & 4 & 11.1 & 19 & 52.8 & 14.37 & $0.001 *$ \\
\hline
\end{tabular}

Significant at level $\mathrm{p}<0.001 * *$

Vol. 12 No. 1 May, 2017 
Tanta Scientific Nursing Journal

Table (7): Percentage distribution of studied children suffering from neutropenia under chemotherapy related to laboratory investigation.

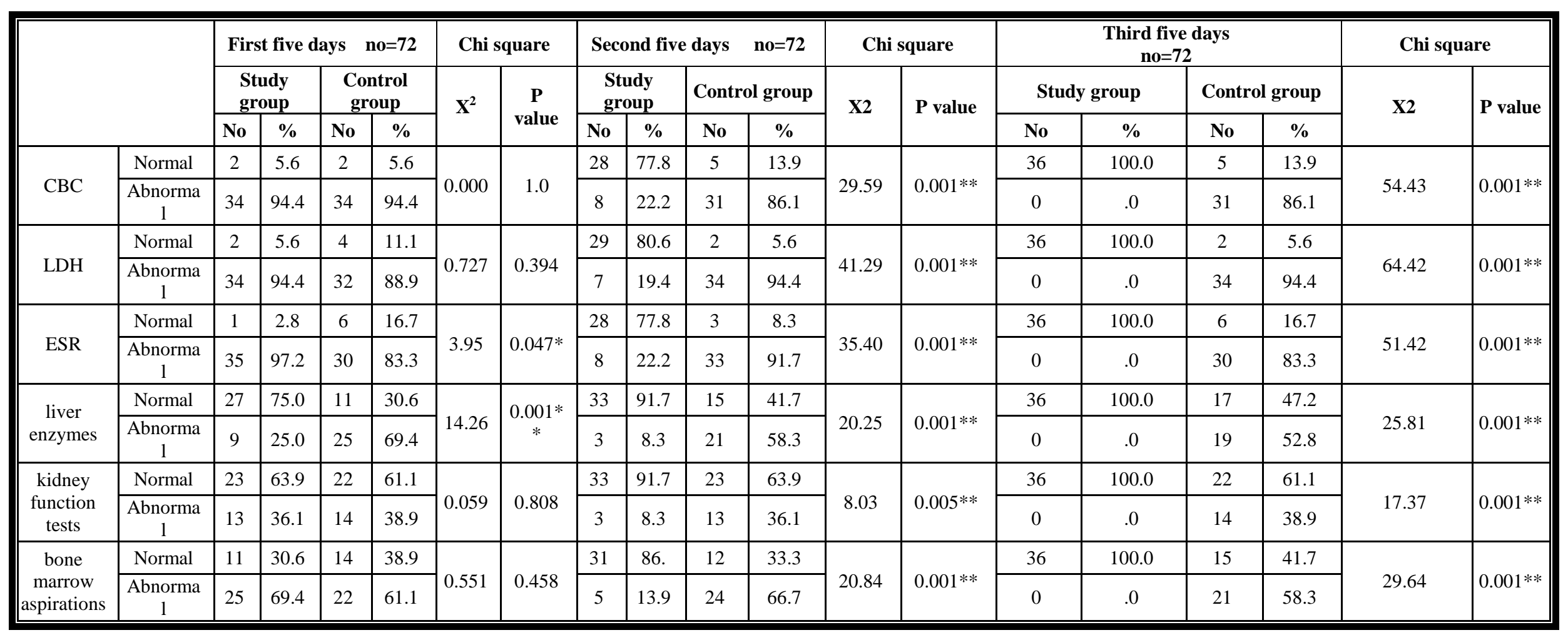

\section{Significant at level $p<0.001 * *$}


Tanta Scientific Nursing Journal

Table (8): Percentage distribution of studied children suffering from neutropenia under chemotherapy related to general complication.

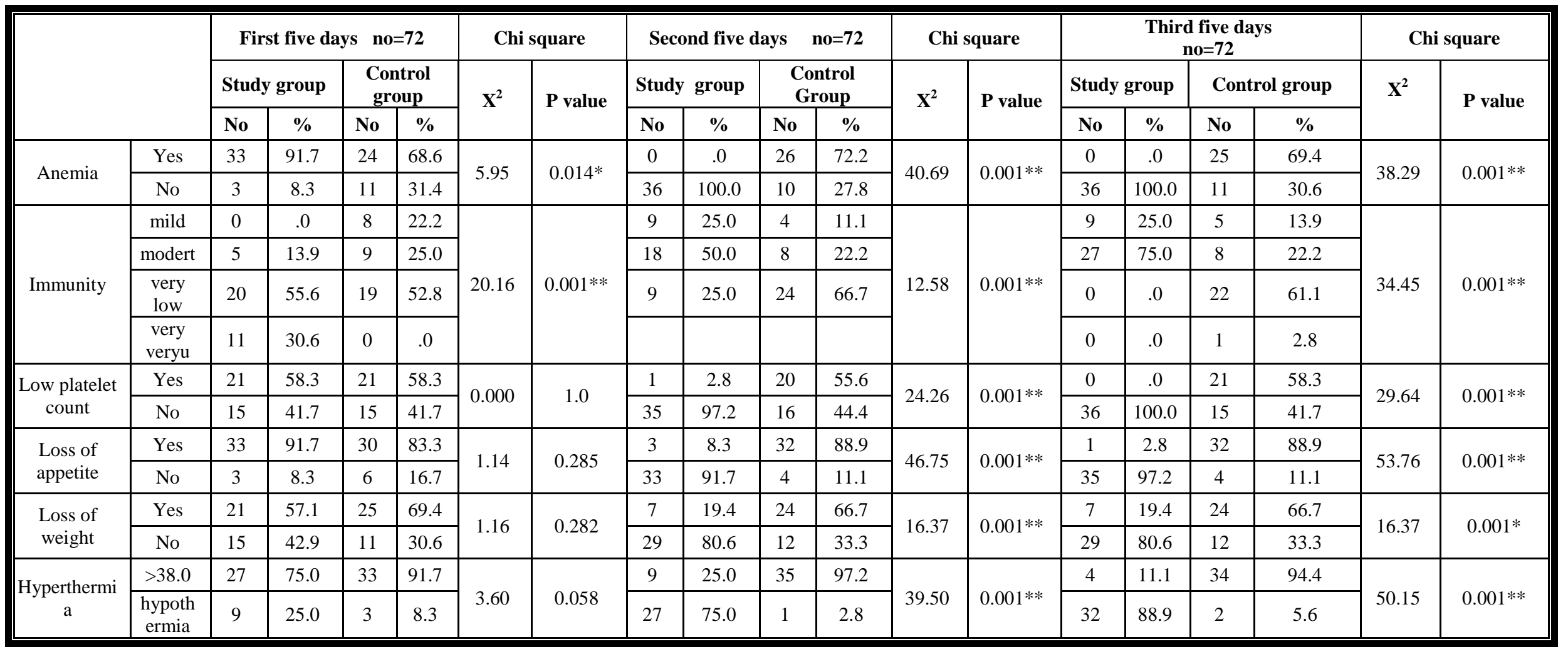

Significant at level $\mathbf{p}<0.001 * *$

Vol. 12 No. 1 May, 2017 
Tanta Scientific Nursing Journal

Table (9): Percentage distribution of studied children suffering from neutropenia under chemotherapy related to specific complication.

\begin{tabular}{|c|c|c|c|c|c|c|c|c|c|c|c|c|c|c|c|c|c|c|c|}
\hline & & \multicolumn{4}{|c|}{ First five days no $=72$} & \multicolumn{2}{|c|}{ Chi square } & \multicolumn{4}{|c|}{ Second five days $n o=72$} & \multicolumn{4}{|c|}{ Chi square } & \multirow{2}{*}{\multicolumn{2}{|c|}{$\begin{array}{c}\text { Third five days } \\
\text { no=72 }\end{array}$}} & \multicolumn{2}{|c|}{ Chi square } \\
\hline & & \multicolumn{2}{|c|}{$\begin{array}{l}\text { Study } \\
\text { group }\end{array}$} & \multicolumn{2}{|c|}{$\begin{array}{l}\text { Control } \\
\text { group }\end{array}$} & \multirow{2}{*}{$X^{2}$} & \multirow{2}{*}{$\begin{array}{c}\mathbf{P} \\
\text { value }\end{array}$} & \multicolumn{2}{|c|}{ Study group } & \multicolumn{2}{|c|}{ Control group } & \multirow{2}{*}{$\mathbf{X}^{2}$} & \multirow{2}{*}{$P$ value } & \multicolumn{2}{|c|}{ Study group } & & & \multirow{2}{*}{$\mathbf{X}^{2}$} & \multirow{2}{*}{$P$ value } \\
\hline & & No & $\%$ & No & $\%$ & & & No & $\%$ & No & $\%$ & & & No & $\%$ & No & $\%$ & & \\
\hline \multirow{2}{*}{$\begin{array}{l}\text { inflammation } \\
\text { at mucous } \\
\text { membrane } \\
\end{array}$} & Yes & 30 & 83.3 & 34 & 94.4 & \multirow[b]{2}{*}{2.25} & \multirow[b]{2}{*}{0.134} & 5 & 13.9 & 34 & 94.4 & \multirow[b]{2}{*}{47.04} & \multirow[b]{2}{*}{$0.001 * *$} & 4 & 11.1 & 34 & 94.4 & \multirow[b]{2}{*}{50.15} & \multirow[b]{2}{*}{$0.001 * *$} \\
\hline & No & 6 & 16.7 & 2 & 5.6 & & & 31 & 86.1 & 2 & 5.6 & & & 32 & 88.9 & 2 & 5.6 & & \\
\hline \multirow{2}{*}{ Vomiting } & Yes & 29 & 80.6 & 33 & 91.7 & \multirow{2}{*}{1.85} & 0173 & 2 & 5.6 & 32 & 88.9 & 5015 & $0001 * *$ & 0 & .0 & 32 & 88.9 & 5760 & $0001 * *$ \\
\hline & No & 7 & 19.4 & 3 & 8.3 & & 0.173 & 34 & 94.4 & 4 & 11.1 & $30.1 J$ & $0.001 \%$ & 36 & 100.0 & 4 & 11.1 & 51.00 & 0.001 \\
\hline Diarrhea & Yes & 20 & 55.6 & 22 & 61.1 & 0232 & 0633 & 6 & 16.7 & 22 & 61.1 & 1469 & $0001 * *$ & 6 & 16.7 & 21 & 58.3 & 1333 & $0001 * *$ \\
\hline Diarrnea & No & 16 & 44.4 & 14 & 38.9 & 0.232 & 0.033 & 30 & 83.3 & 14 & 38.9 & 14.09 & $0.001 \%$ & 30 & 83.3 & 15 & 41.7 & 13.33 & $0.001 \cdots$ \\
\hline locs of hair & Yes & 24 & 66.7 & 27 & 75.0 & 0605 & 0437 & 22 & 61.1 & 26 & 72.2 & 100 & 0317 & 23 & 63.9 & 26 & 72.2 & 0575 & 0448 \\
\hline Ioss or nair & No & 12 & 33.3 & 9 & 25.0 & 0.005 & 0.431 & 14 & 38.9 & 10 & 27.8 & 1.00 & 0.317 & 13 & 36.1 & 10 & 27.8 & 0.315 & 0.448 \\
\hline & Normal & 8 & 22.2 & 3 & 8.6 & & & 25 & 69.4 & 6 & 16.7 & & & 25 & 69.4 & 6 & 16.7 & & \\
\hline Neurological & Headache & 28 & 77.8 & 31 & 88.6 & 3.41 & 0.182 & 11 & 30.6 & 29 & 80.6 & 20.74 & $0.001 * *$ & 11 & 30.6 & 29 & 80.6 & 20.74 & $0.001 * *$ \\
\hline & Paralysis & 0 & .0 & 1 & 2.9 & & & 0 & .0 & 1 & 2.8 & & & 0 & .0 & 1 & 2.8 & & \\
\hline & Normal & 12 & 33.3 & 3 & 8.3 & & & 27 & 75.0 & 1 & 2.8 & & & 30 & 83.3 & 1 & 2.8 & & \\
\hline skeletal & Pain & 12 & 33.3 & 23 & 63.9 & 9.038 & $0.011 *$ & 5 & 13.9 & 25 & 69.4 & 40.04 & $0.001 * *$ & 6 & 16.7 & 24 & 66.7 & 48.92 & $0.001 * *$ \\
\hline system & $\begin{array}{l}\text { pain during } \\
\text { examination }\end{array}$ & 12 & 33.3 & 10 & 27.8 & & & 4 & 11.1 & 10 & 27.8 & & & 0 & .0 & 11 & 30.6 & & \\
\hline Bleeding & Yes & 24 & 66.7 & 11 & 30.6 & 039 & $0.002 *$ & 11 & 30.6 & 12 & 33.3 & 0.064 & 0801 & 5 & 13.9 & 12 & 33.3 & 377 & 0052 \\
\hline Bleeaing & No & 12 & 33.3 & 25 & 69.4 & 9.59 & $*$ & 25 & 69.4 & 24 & 66.7 & 0.004 & 0.801 & 31 & 86.1 & 24 & 66.7 & 0.11 & .052 \\
\hline level of & Normal & 3 & 8.3 & 7 & 19.4 & & & 28 & 77.8 & 3 & 8.3 & & & 29 & 80.6 & 3 & 8.3 & & \\
\hline activity & Abnormal & 33 & 91.7 & 29 & 80.6 & 1.85 & $0.1 / 3$ & 8 & 22.2 & 33 & 91.7 & 35.40 & $0.001 \cdots$ & 7 & 19.4 & 33 & 91.7 & 38.02 & $0.001 \cdots$ \\
\hline & Normal & 8 & 22.2 & 3 & 8.3 & & & 35 & 97.2 & 3 & 8.3 & & & 36 & 100.0 & 3 & 8.3 & & \\
\hline system & Abnormal & 28 & 77.8 & 33 & 91.7 & 2.68 & 0.101 & 1 & 2.8 & 33 & 91.7 & 57.06 & $0.001^{* *}$ & 0 & .0 & 33 & 91.7 & 60.92 & $0.001^{* *}$ \\
\hline & Rapid & 32 & 88.9 & 31 & 86.1 & & & 6 & 16.7 & 33 & 91.7 & & & 0 & .0 & 32 & 88.9 & & \\
\hline $\begin{array}{l}\text { respiratory } \\
\text { rate }\end{array}$ & Slow & 4 & 11.1 & 5 & 13.9 & 0.127 & 0.722 & 14 & 38.9 & 3 & 8.3 & 41.80 & $0.001 * *$ & 0 & .0 & 4 & 11.1 & 72.00 & $0.001 * *$ \\
\hline & Normal & 0 & .0 & 0 & .0 & & & 16 & 44.4 & 0 & .0 & & & 36 & 100.0 & 0 & $.0 \%$ & & \\
\hline
\end{tabular}

\section{Significant at level $\mathbf{p}<0.001 * *$}

Vol. 12 No. 1 May, 2017 


\section{Discussion}

As regards to biosocial characteristics, the present study showed that, about mean age of nine years of studied children was between 6-12 years. This result is in disagreement with Legg et al. (2004) who found that the long-term incidence rates and trends (1975 to 2004) are adjusted for delays in reporting where possible. Delayed reporting primarily affects the most recent 1 to 3 years of incidence data (in this case, 2002 to 2004), especially for cancers such as melanoma, leukemia, and prostate that are frequently diagnosed in outpatient settings. ${ }^{(179)}$

Moreover, the male to female incidence in this study was exceed than female. This was in accordance with those of Zelzar, (2000), who pointed out that cancer, is more prevalent among male agreements with that study American Cancer Society's Cancer Statistics Center (2012) cancer is slightly more common among Hispanic and white children than among African-American and Asian-American children, and it is more common in boys than in girls. AML occurs about equally among boys and girls of all races. ${ }^{(33,180)}$

However no statistical significant difference was detected between both study and control groups of children regarding to sex.This study this result is in disagreement with Fritz et al. (2008)cancer incidence rates were stable in males from 1995 to 2008 and in females from 1999 to 2008, female exceed more than male. ${ }^{(181)}$

Regarding to educational level, the present study relieved that most of children with cancer at primary school, this result disagreements with Ferlay et al. (2010), cancer is being diagnosed more frequently during the child-rearing years. Sociodemographic and cancer-related information on families and minor (0-18 years). ${ }^{(182)}$ Most of them were primary school in study group compared to control group were preparatory school. As regarding to setting it was equal percentage. So this table shows that there was no statistically significant difference between study and control group of children suffering from neutropenia under chemotherapy in relation to their age, sex, setting but level of education most of them were primary school.

Regarding to diagnosis, the present study relieved that most of children would have leukemia of study group and less of them having solid tumors of control group, this result was in accordance with those of American Cancer Society's Cancer Statistics Center, (2012). ${ }^{(33)}$, Leukemia is the most common cancer in children $70 \%$ of children and teens, accounting for almost 1 out of 3 cancers. Overall, peaking between 2 and 4 years of age. Cases of AML are more spread out across the 
childhood years, but this type of leukemia is slightly more common during the first 2 years of life and during the teenage years. This result disagreement with my study Raab, (2009), in general, cancer in children and teenagers is uncommon, accounting for less than $1 \%$ of all cancer cases in the United States. This year, an estimated 10,380 children younger than 15 and about 5,000 adolescents aged 15 to 19 will be diagnosed with cancer in the United States. This study agreement with this research Mitchell et al. (2009, solid tumors make up about $30 \%$ of all pediatric cancers. The most common types of solid tumors in children include brain tumors, neuroblastoma, rhabdomyosarcoma,

Wilms' tumor, and osteosarcoma. ${ }^{(45,46)) \text {, }}$

The present study indicated that the disease process had effect on children educational level, most of children wasn't attended in study group of the studied children in both study and control group did not attended at the school. As well as the absenteeism rate was high, majority of children were absent school. However there was no statistical difference between the study and control group. This could by due to the fact. Those children have gone to hospital to receive their medication and bad psychological state, as illustrated for the reason of absenteeism. More than half of the studied children due to hospital admission. The result of the present study contrast the study performed for children with cancer in royal hospital service and $\mathrm{Al}$ Amire Rahme Hospital in Jordan (Holloway, 2009) ${ }^{(183)}$.

The result of the present study agree with the result of Polovich et al. (2009) .who explained that children with cancer have to received chemotherapy in a regular basis and Gharaibeh, (2009) ' reported that education was one of greatest difficulties affected children with cancer. Similarly, Abd-El Baset, (2003)' mentioned that the education of two third of children with cancer at school age was influenced by their attendance. ${ }^{(67,184,185)}$,

Concerning hereditary and maternal history for cancer. The result of the present study was found that, $14 \%$ of study group having hereditary factors to cancer in children compared to $80.6 \%$ of control group have no hereditary factors to cancer in children. This study was disagreemements with Goldgar et al. (2010), both environmental and hereditary factors cause cancer. Studies of familial cancer aggregation have been the main approach in the assessment of the hereditary effects in cancer. ${ }^{(186)}$,

El-Nagar, (2005) who stated that there is a broad consensus on the predominant importance of environmental factors and somatic events in human cancer agree that some $60-90 \%$ of the studied cancers can be explained by environmental factors 


\section{Tanta Scientific Nursing Journal}

only. Not shared among family members ${ }^{*}(187)$

As regarding to daily activity, level of activity and physical examination at first five days, the current study showed that no statistically significant differences between two groups, the two groups not active, no play, and physical examination having many abnormalities, at second five days the current study showed that the improvement for children and the differences between two groups after implementation of clinical pathway there were highly statistically significant differences between two groups in second five days the children in study group become normal active, play in calme way and all physical examination were improvement for the study group, rather than the control group who received the routine hospital care finally the third five days all children in study group hyper active, play in a groups in gardens and the physical assessment differentiated after implementation of clinical pathway so there were a highly statistically significant differences between two groups

The result of the present study in accordance with Gharaibeh, (2009) who stated that the clinical pathway has direct and tangible impact on children care process and outcome for children with cancer under chemotherapy suffering from neutropenia. (184),

The child didn't play until recovered from neutropenia, neutropenia affect the psychological condition of the child which they prefers isolation during neutropenia. So application of clinical pathway assist them to reduce the length of time of neutropenia for return to normal condition. The present study revealed that the clinical pathway implemented by the researcher with the hospital team had effect on the studied children in relation to physical assessment, face, eye, lips, gum, skin, gastrointestinal problems and skeletal system problem. Which all physical assessment improved after implementation of clinical pathway in first five days and second five days and reach to normal at second five days so their were a statistically significant deference between study group at first five days and second five day after implementation of clinical pathway and reached to a highly statistically significant deference between study group at first five days and third five day.

The recovery of children from neutropenia is gradual and take time to occur not sudden. The role of clinical pathway good quality of care in fewer times. Also used to minimize average length of stay without compromising the quality of care process and out come.

The finding was in accordance with the result, Zezler, (2000), who mentioned that 
clinical pathway had effect on finding of the current study revealed that there was a highly statistical significant in physical assessment. There was a prognosis in the result as well as face, eye, lips, gum, skin, gastrointestinal problems and skeletal system problem. ${ }^{(180),}$

Concerning the effect of clinical pathway for the study groups of the responding of children to daily nursing care, our study revealed that their was statistically significant difference between study groups of children in relation to their response to daily nursing care, in first five days and second five days the response of children was fifty percent related to daily weight, monitor of urine out put, fever, paller, infection, pain, subsidetofiftypercent. Hematourea, tenderness, purbra, mucositis subside to hundred percent.

Concerning the effect of clinical pathway for the study groups of the responding of children to daily nursing care at third five days their was statistically significant difference between study groups of children in relation to their response to daily nursing care, their response to daily nursing care reached to hundred percent.

That study is in agreement with Rekha, (2005), who stated that Clinical pathway is a tool used in achieving coordinated care and desired outcomes within an anticipated time frame by utilizing the appropriate resources available. A clinical pathway is a blueprint that guides the clinician in the provision of care ${ }^{.(192), \text {. }}$

The present study revealed that the clinical pathway implemented by the researcher with the hospital team had effect on the studied children in relation to their nutritional habits and effect in reducing their problems and their response to medication, their was a statistically significant differences between study groups of children which all feeding habits improved at first five days then reached to normal at third five days. The number of meal increased from one to two meals and reached to three beer day, their appetite improved, all gastrointestinal problems disappear along fifteen days. (192),.

Regarding to their response to medication in study groups of children their response to medication different from first five days rather than second five days, they improved on treatment and reached to normal in third five days.

the child when he admitted to hospital in neutropenia theses children is in a very bad general condition, is in needed emergency care and treatment include isolation, blood transfusion, intravenous fluid and antibiotic means high quality of care, the all team conceder this child is critical case. All team acting for saving his life.

That study is in agreements with Polit, (2009) who found that interventional 
categories consist of groups of activities that make up a comprehensive treatment, these categories typically include, tests, treatments and nursing interventions, consultations, medications, diet, activity, child and family education, and discharge planning. Outcomes focus on child care activity and nutrition are defined for each time interval, realistic, reflect incremental progress, and achievable by $90 \%$ of the children. ${ }^{(193),}$

\section{Conclusion}

The study could be concluded that:

Based on the results of the present study it could be concluded that Children with cancer suffering from neutropenia undergoing chemotherapy, to whom clinical pathway was applied had fewer complications, less readmission after discharge compared with those who received routine hospital care.

\section{Recommendations}

Based on the findings of the current study, the following recommendation is needed:

- Application of clinical pathway for children suffering from neutropenia undergoing chemotherapy was essential for reducing complications and less readmission.

- Clinical pathway should be applied as a routine nursing care for children with neutropenia undergoing chemotherapy. Hospitals should establish a policy concerning clinical pathway that should be available to each hospital units.

- Further studies are needed in order to apply and demonstrate the research on a larger population for generalization of results.

- Establish training for medical and paramedical staff regarding application of clinical pathway.

\section{Future recommendation:}

- A future study can be conducted for large sample

- Establishing a health education unit in the hospital to provide health education sessions for all heath team member and children.

\section{References}

1. Higgins J, Green S. Cochrane Handbook for Systematic Reviews of Interventions. J Nurs Manag 2009; 14 (1):529-537.

2. Murphy S, Kochanek K. Deaths: Final Data for 2010. National Vital Statistics Reports. National Center for Health Statistics 2013;61(4):22-32.

3. Steliarova E, Stiller C, Kaatsch P. Geographical patterns and time trends of cancer incidence and survival among children and adolescents in Europe since the 1970s: an epidemiological study. J Lancet 2004; 364(1):2097-2105. 
4. swedishChildhoodCancerFoundationB arncancerfonden].Availableat http://barn.barncancerfonden.se/Behall are/Tidning/Tidigarenummer/2001/Ba rnCancer401/Artiklar/Overlevnadsstati stik.on October 12013.

5. Milne E, Greenop K, Metayer C. Fetal growth and childhood acute lymphoblastic leukemia. findings from the childhood leukemia international consortium. Int J Cancer 2013; 133 (2):2968-2979.

6. National Cancer Institute. A Snapshot of Pediatric Cancers. 2014. Available at www. cancer. gov/ researchand funding/ snapshots/pediatric on November 72014.

7. Weingart S, Brown E, Bach P. NCCN Task Force Report: Oral chemotherapy. J Natl Compr Canc Netw 2008; 6(3): 1-14.

8. Kuderer N, Cosler L, Crawford J, Dale D, Lyman G. Cost and mortality associated with febrile neutropenia in adult cancer patients. Proc Am Soc Clin Oncol 2002; (1):21: 250.

9. Dees E, O'Reilly S, Goodman S. A prospective pharmacologic evaluation of age-related toxicity of adjuvant chemotherapy in women with breast cancer j Cancer Invest 2000; 18(1): 521-529.

10. Gandhi S, Arguelles L, Boyer J. Economic impact of neutropenia and febrile neutropenia in breast cancer: estimates from two national databases. Pharmacotherapy 2001; 21(1): 684 690.

11. Rotter T, Koch R, Kugler J, Gothe H, Kinsman L, James E. Clinical pathways: effects on professional practice, patient outcomes, length of stay and hospital costs. Cochrane Database Syst Rev 2007, 3:CD006632

12. Vanhaecht K, Witte K, Depreitere R, Sermeus W. Clinical pathway audit tool: a systematic review. J Nurs Manag 2006; 14 (1):529-537.

13. Campbell H, Bradshaw N, Porteous M. Integrated care pathways. J Of Pediatric 2010; 316(1):133-144.

14. Legg L, Feuer E, Midthune D, et al. Impact of reporting delay and reporting error on cancer incidence rates and trends. J Natl Cancer Inst 2002; 94(2): 1537-1545.

15. American Cancer Society. Global Cancer Facts \& Figures 2nd Edition. Atlanta: American Cancer Society 2012;105-133.

16. Zelzar L. Children's memories for painful treatment procedures. Journal of National Caner Institute, 2000; $6(1): 4$

17. Fritz A, Percy C, Jack A. International Classification of Diseases for Oncology, 3rd ed. Geneva, Switzerland: World Health Organization; 2008. 
18. Raab C, Gartner J. Diagnosis of childhood cancer. Prim Care 2009; 36(4):671- 684

19. Mitchell C, Hall G, Clarke R. Acute leukemia in children: diagnosis and management, BM J 2009; 338(1):2285.

20. Holloway D. The role of clinical pathways in improving patient out comes. Journal of National Cancer Institute: New York, 2009; 102(2):105.

21. Gharaibeh H. The psychological burden of patients with hemolytic anemia. J Pediatric Int: 2009; 51(2):630-636

22. Abd-El Baset A, Ghashaba A and Morsy M. Orem's Self Care Model: A medication leaflet for the mothers whose children are suffering from bronchial asthma. Master Thesis, Faculty of Nursing, Ain Shams University, 2003; 20-22.

23. Polovich M, White J, Olsen M. Chemotherapy and Biotherapy Guidelines and Recommendations for Practice (Oncology Nursing Society, Pittsburgh. PA,3ed;2009.

24. Goldgar D, Easton D, CannonAlbright L, Skolnick M. Systematic population-based assessment of cancer risk in first-degree relatives of cancer probands. J Natl Cancer 2010; 86(2): 1600-7.
25. El-Naggar M. Short Altars in paediatrics. University Book Center, E 2005.

26. Gharaibeh H. The psychological burden of patients with hemolytic anemia. J Pediatric Int: 2009; 51(2):630-636.

27. Zelzar L. Children's memories for painful treatment procedures. Journal of National Caner Institute, 2000; $6(1): 4$.

28. Rekha J. What is a clinical pathway? Development of a Definition to Inform the Debate. (4th ed.,). Churchill, Livingstone Company: London, 2005; $71-72$.

29. Polite R. Hemolytic Anemia. Practical Hematology. (9th ed.,). United Kingdom Company: London, New York, 2009; 32-33.

30. Virginia M and Lisa V. Home health care management and practice, using outcome. Based Critical Pathways to Improve Documentation, 2010; 8:6.

31. Rekha J. What is a clinical pathway? Development of a Definition to Inform the Debate. (4th ed.,). Churchill, Livingstone Company: London, 2005; 71-72.

32. Polite R. Hemolytic Anemia. Practical Hematology. (9th ed.,). United Kingdom Company: London, New York2009;32-33 